\section{Assessment of Adherence of Diagnostic Accuracy Studies Published in Radiology Journals to STARD Statement Indexed in Web of Science, PubMed \& Scopus in 2015}

\author{
Zarei F., ${ }^{1,2}$ Zeinali-Rafsanjani B. ${ }^{2 *}$
}

\begin{abstract}
Rationale and Objective: The objective of this study is to evaluate the methodological adherence of diagnostic accuracy studies published in radiology journals, which were indexed in different databases with the STARD standard guide 2015.

Materials and Methods: The different databases were searched in order to find suitable journals. Among 84 English radiology journals, 31 journal were selected randomly. In order to find the articles, the same search fields and search terms were used. All the items of STARD checklist 2015 were considered to take in to account in assessment of the adherence of the articles to the standard. Total STARD score for each article was calculated by summing the number of reported items.

Results: 151 articles from 31 journals were evaluated to check the adherence of their structure to STARD standard. Based on the results the articles had the most adherence with the STARD standard in material and method part the item of participants, discussion section, and title or abstract. On the contrary, most of the articles were not adhere to other information which are new items in STARD 2015. Among radiology diagnostic accuracy articles only one article $(0.66 \%)$ had a registration number and $10(6.62 \%)$ articles had a link to full study protocol. More than $60 \%$ of articles adhered to the ethics (69.54\%) and source of support (63.58\%).
\end{abstract}

Conclusions: The radiology diagnostic accuracy studies were adhered to $69.45 \%$ STARD items, which shows an improvement in reporting the diagnostic accuracy articles in comparison to previous studies.

Keywords

STARD Statement, Diagnostic Accuracy, Adherence

\section{Introduction}

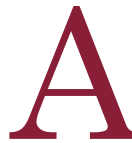

diagnostic accuracy study is a research which prepares a proof on how well a test or a special procedure accurately recognizes or precludes diseases. This type of study helps clinicians and healthcare suppliers regard the choice of a suitable management method for each patient [1]. A comprehensive and accurate reporting of diagnostic accuracy studies is essential to permit readers to evaluate any possible bias in the study and to assess the reproducibility of the results. Accordingly, in order to compare different diagnostic methods, there should be a standard procedure for perform the study and report the results so as to avoid exaggerated conclusion and make the results gen-
${ }^{1}$ Department of Medical Journalism, School of

Para-Medicine, Shiraz

University of Medical Sci-

ences, Shiraz, Iran

${ }^{2}$ Medical Imaging Re-

search Center, Shiraz

University of Medical

Sciences, Shiraz, Iran

*Corresponding author: B. Zeinali-Rafsanjani Medical Imaging Research Center, Shiraz University of Medical Sciences, Shiraz, Iran E-mail: B.zeinali.r@ gmail.com

Received: 22 July 2017 Accepted: 15 October 2017 
eralizable [1].

A group of scientists established STARD (Standards for Reporting Diagnostic Accuracy) statement to improve the quality of reporting these studies. At first, STARD statement consisted of a checklist of 25 items and a flow diagram in which authors can ensure that all relevant information is present [2]. This standard covers the title, abstract, keywords (Item 1), introduction (Item 2), methods (Items 3-13), results (Items14-24) and discussion (Item 25). In the method section, the required information regarding the participants (Items 3-6), test methods (Items 7-11), and statistical methods (Items 12-13) were described in detail. The required information under result section regarding participants (Items 14-16), test results (Items 17-20) and estimates (Items 21-24) were precisely explained.

This checklist was reviewed in 2015, and its items increased to 30 [3]. This revision is intended to simplify the application, understanding and distribution of the checklist. The new document [3] comprises an explanation of the definition, rationale and optimal usage of each element on the checklist, as well as a brief summary of the existing evidence on bias and applicability. The new standard also covers the title (Item 1), abstract (Item 2), introduction (Items 3-4), methods (Items 5-18), results (Items 19-25) and discussion (Items 26-27); it also requires new information (Items 28-30). In the method section, the required information about the study design (Item 5), participants (Items 6-9), test methods (Items 10-13) and analyses (Items 14-18) are described in detail. Even some items are split into 2 items such as item 10 (requiring the information about the index and reference tests), item 12 (the cutoffs of reference and index test) and item 13 (the availability of reference and index test). The required information in result section about participants (Items 19-22) and test results (Items 23-25) were precisely explained. Item 21 , which is about the distribution of the disease and the distribution of alter- native diagnosis is split into 2 parts.

Previously, some studies evaluated the effectivity of STARD statement and how well the diagnostic accuracy articles adhered to this standard. Some studies assessed the papers published in specific fields such as ophthalmology, gastrointestinal disease, obstetric and gynecology, musculoskeletal disorders and hepatology. Most of these articles reported a non-desirable adherence of articles to the standard leading to fewer effects of this standard on improving the diagnostic accuracy reports [4-18].

To the best of our knowledge, there are no studies on the adherence of radiology and medical imaging articles to the STARD statement, although there are lots of radiology diagnostic accuracy studies.

The objective of this study is to evaluate the adherence of diagnostic accuracy studies published in 2015 in the English language radiology journals which were indexed in the Web of Science, PubMed and Scopus with STARD standard guide 2015 .

\section{Material and Methods}

This was a cross-sectional study that evaluated 151 diagnostic accuracy articles published in radiology journals indexed in the Web of Science, PubMed and Scopus databases. The sample size was determined based on 131 articles using formula 1 [19]:

$$
n=Z_{1-\alpha / 2}^{2} P(1-P) / d^{2} \quad \text { Eq. } 1
$$

Where, $\mathrm{n}=$ sample size, $Z_{1-\alpha / 2}^{2}=$ confidence interval, $\mathrm{P}=$ estimated proportion, $\mathrm{d}=$ desired precision.

\section{Sampling}

The sampling was done as follows:

1. Firstly, the Web of Science, Scopus and PubMed databases were searched in order to find journals that contain diagnostic accuracy articles. Accordingly, to find such journals, an advanced search was performed. In the web- 
site of the web of science, the search fields of title, document type and the topic were used. The keywords and search fields were used in different databases. The keywords were selected in the way to narrow down the search field and to obtain possible results too (Table 1). Additional filters such as subject area and document type were also used after obtaining the results. This search led to collecting 84 radiology journals which contained diagnostic accuracy articles.

2. In order to randomize the obtained list of journals, an online randomizer was used (http://www.randomizelist.com).

3. In order to select 31 English Radiology Journals out of 84 journals, an online random number generator was used (https://www.random.org). Since some Journals did not contain enough number of diagnostic accuracy articles, one more journal was selected randomly so that the overall number of selected journals hit 31 .

4. In order to find the articles from each jour- nal, the same search fields and search terms as "Table 1" were used; the only difference in this step was that the given journal was selected to limit the search to a specific journal. Totally, 275 articles were selected (Figure 1).

5. Among selected articles, 151 articles were randomly selected to evaluate the adherence of their structures to STARD statements (Figure 1).

\section{Assessment of STARD Checklist}

Although the latest version of STARD checklist [3] was introduced in 2015, since there was not much difference between this and earlier version (just 3 new items were added) and moreover because of its better categorization of different items (splitting some previous items into 2 parts), it was used to take into account in assessment the adherence of the articles published in 2015 to this standard.

It is also assessed that if the word of "STARD" standard was pointed out in the studies or not? The reported items were scored 1 and not

Table 1: Search fields and search terms used in different databases

\begin{tabular}{|c|c|c|c|c|c|}
\hline \multirow[t]{2}{*}{ Database } & \multicolumn{5}{|c|}{ Search fields } \\
\hline & Title & Abstract & Keyword/Mesh & Source title & Topic \\
\hline Web of Science & $\begin{array}{c}\text { Diagnosis OR } \\
\text { diagnostic OR } \\
\text { accuracy OR } \\
\text { sensitivity OR } \\
\text { specificity OR } \\
\text { efficacy OR } \\
\text { Compare }\end{array}$ & -------- & -------- & ------ & $\begin{array}{c}\text { Radiology and } \\
\text { nuclear medicine }\end{array}$ \\
\hline Scopus & $\begin{array}{c}\text { Diagnosis OR } \\
\text { diagnostic OR } \\
\text { accuracy OR } \\
\text { sensitivity OR } \\
\text { specificity OR } \\
\text { efficacy OR } \\
\text { Compare }\end{array}$ & $\begin{array}{c}\text { Diagnosis OR } \\
\text { diagnostic OR } \\
\text { accuracy OR } \\
\text { sensitivity OR } \\
\text { specificity OR } \\
\text { efficacy OR } \\
\text { Compare }\end{array}$ & $\begin{array}{c}\text { Diagnosis OR } \\
\text { diagnostic OR } \\
\text { accuracy OR } \\
\text { sensitivity OR } \\
\text { specificity OR } \\
\text { efficacy OR } \\
\text { Compare }\end{array}$ & $\begin{array}{c}\text { Radiology OR } \\
\text { imaging OR } \\
\text { ultrasound }\end{array}$ & $\begin{array}{c}\text { Radiology and } \\
\text { nuclear medicine }\end{array}$ \\
\hline PubMed & $\begin{array}{c}\text { Diagnosis AND } \\
\text { diagnostic AND } \\
\text { accuracy AND } \\
\text { sensitivity AND } \\
\text { specificity AND } \\
\text { efficacy }\end{array}$ & --------- & --------- & ------- & \\
\hline
\end{tabular}




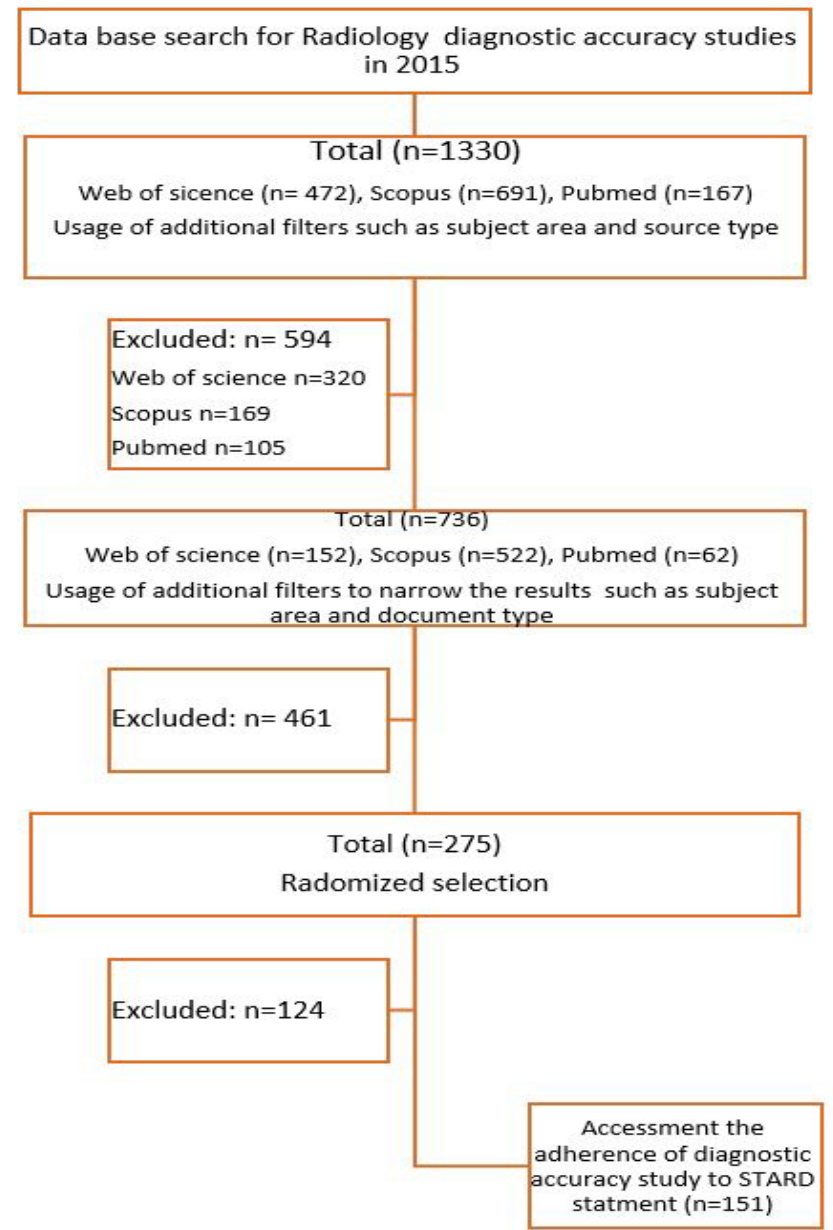

Figure 1: Flow chart showing the search results and limitation the results.

reported ones were scored 0 . The items that were considered in each section were shown in below tables. Items 1 and 18 were split into 2 sections. Items $1 \mathrm{a}$ and $1 \mathrm{~b}$ were about the presence of the diagnostic accuracy terms in title and abstract respectively. Item $18 \mathrm{a}$ and $18 \mathrm{~b}$ were scored the presence of pre-specifying the sample size and how the samples were collected respectively. We also scored the jour- nals which introduce the STARD statement in instructions for authors. The items of STARD statement that assessed in this study was illustrated in Tables 2-5.

\section{Searching the Text}

In order to accelerate the searching inside the text of articles, some keywords were used. For instance, in order to assess the items of title

Table 2: The items which were assessed in selected diagnostic accuracy articles to check the adherence of title and abstract section to the STARD. It also assessed if the STARD standard was pointed out in the studies or not. 
Table 3: The items which were assessed in selected diagnostic accuracy articles to check the adherence of method section to the STARD. Item \#18 was broken into two sections: 1 . If the sample size was pre-specified. 2 . How the samples were chosen?

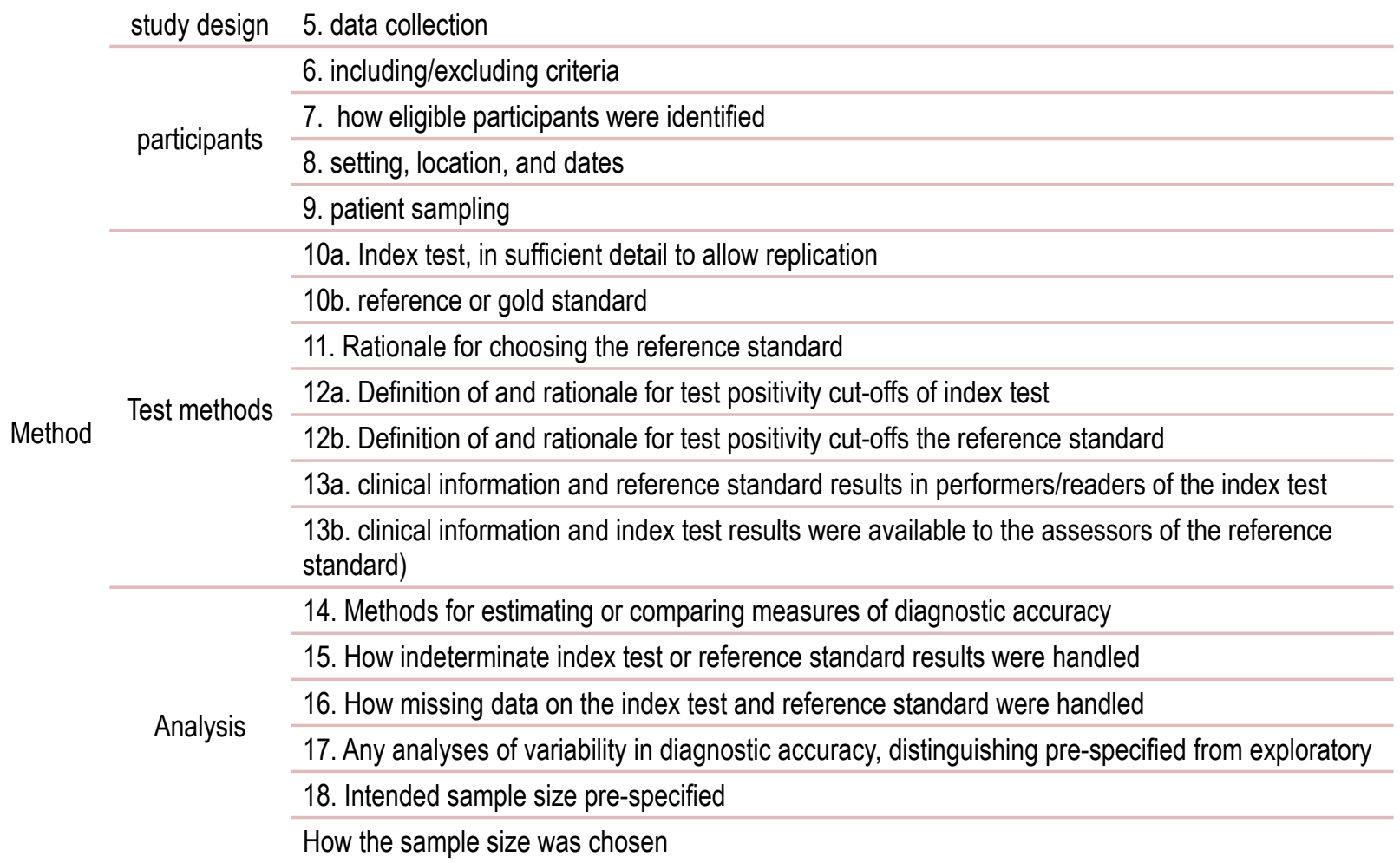

Table 4: The items which were assessed in selected diagnostic accuracy articles to check the adherence of result section to the STARD

19. Flow of participants, using a diagram

20. Baseline demographic and clinical characteristics of participants

Participants 21a. Distribution of severity of disease in those with the target condition

$21 \mathrm{~b}$. Distribution of alternative diagnoses in those without the target condition

22. Time interval and any clinical interventions between index test and reference standard

Results

23. Cross tabulation of the index test results (or their distribution) by the results of the reference standard

Test results

24a. Estimates of diagnostic accuracy (accuracy, area under curve or ROC)

$24 \mathrm{~b}$. Estimates of diagnostic accuracy and their precision (such as $95 \%$ confidence inter-

vals, SD, Standard error)

25. Any adverse events from performing the index test or the reference standard 
Table 5: The items which were assessed in selected diagnostic accuracy articles to check the adherence of discussion section and other information to the STARD

\begin{tabular}{ll} 
DISCUSSION & \begin{tabular}{l} 
26. Study limitations, including sources of potential bias, statistical uncertainty, and generalizability \\
\cline { 2 - 2 } OTHER INFORMATION Implications for practice, including the intended use and clinical role of the index test
\end{tabular} \\
\hline & $\begin{array}{l}\text { 28. Registration number and name of registry } \\
\text { 29. Where the full study protocol can be accessed }\end{array}$ \\
\hline 30. Sources of funding and other support; role of funders (conflict of interest)
\end{tabular}

and abstract the keywords such as "diagnostic accuracy", "sensitivity and specificity", "efficacy" were used. The related text was found and read to evaluate the item. If the item could not suitably assess by this method the whole text of article was read. The applied keywords for each case was shown in Table 6.

\section{Ethical Considerations}

Because there was no need to work with patients or animals, it was not necessary to get the approval of ethics committee. But the confidentiality of the authors and journals were considered.

\section{Statistical Analysis}

Microsoft Excel 2007 was used to analyze the data. The reported items were scored 1 and not reported ones were scored 0 .

\section{Results}

\section{Randomized Sampling of Journals}

The characteristics of journals which were chosen randomly are shown in Table 7 . The information of $\mathrm{H}$-index and the country was obtained from SJR website. Moreover, it was assessed that if STARD standard was introduced in instruction for authors or not. Besides, the number and percentage of journals from each country and continent were assessed.

The number and percentage of journals which were randomly selected in terms of the country were shown in Table 8.

The number of primarily selected articles and the number of diagnostic accuracy articles which were evaluated has been indicated in Table 9.

Adherence of Articles to STARD Statement

The adherence of 151 radiology diagnostic accuracy studies to STARD 2015 are shown in Table 10.

151 articles (out of 275) from 31 journals (out of 84) were evaluated to check the adherence of their structure to STARD standard.

According to the results radiology articles adhered to $69.45 \%$ of the STARD items. Based on these results, the articles had the most adherence to the STARD standard in title or abstract (97.88\%), material and method part, the item of participants $(96.68 \%)$ and discussion section $(93.05 \%)$. This means that most diagnostic accuracy articles describe the data collection methods, inclusion and exclusion criteria, location, method and date of data sampling. Most of these articles also described the study limitations $(86.09 \%)$.

On the contrary, most articles (64.9\%) did not adhere to "other information" which is a new item in STARD 2015. This item is intended to make the authors add some information such as registration number, a link to the location where the full study protocol can be accessed, sources of any support and ethics.

Among radiology diagnostic accuracy articles only one article $(0.66 \%)$ had a registration number and $10(6.62 \%)$ articles had links to the full study protocol. One of the reasons of this low adherence is that these items were not applicable to radiology diagnostic accura- 
Table 6: The applied keywords for each section of the articles

\begin{tabular}{|c|c|c|c|}
\hline $\begin{array}{c}\text { Section } \\
\text { head }\end{array}$ & Subsection & $\begin{array}{c}\text { Item } \\
\text { number }\end{array}$ & Keywords \\
\hline \multirow{5}{*}{$\begin{array}{c}\text { TITLE OR } \\
\text { ABSTRACT }\end{array}$} & \multirow{5}{*}{ title or abstract } & $1 \mathrm{a}$ & \multirow{5}{*}{ "diagnostic accuracy", "sensitivity and specificity", "efficacy" } \\
\hline & & $1 b$ & \\
\hline & & 2 & \\
\hline & & 3 & \\
\hline & & 4 & \\
\hline \multirow{14}{*}{ Method } & study design & 5 & "retrospective", "prospective" \\
\hline & \multirow{3}{*}{ participants } & 6 & "include", "exclude" \\
\hline & & 7 & "inclusion criteria", "exclusion criteria" \\
\hline & & 9 & "random", Consecutive" \\
\hline & \multirow{7}{*}{ Test methods } & $10 \mathrm{~b}$ & "gold standard", "reference standard", "standard" \\
\hline & & $12 a$ & cut-off \\
\hline & & $12 b$ & cut-off \\
\hline & & $13 a$ & "blind", "mask", "unaware", "knowledge", "observer", "reader" \\
\hline & & $13 b$ & blind, "mask", "unaware", "knowledge", "observer", "reader" \\
\hline & & 14 & "confidence interval" \\
\hline & & 15 & "false", "negative", "positive", "predictive value" \\
\hline & \multirow{3}{*}{ Analysis } & 16 & "miss" \\
\hline & & 17 & "subgroup" \\
\hline & & 20 & "years", "male", "female" \\
\hline \multirow{6}{*}{ Results } & \multirow{3}{*}{ Participants } & $21 a$ & "stage", "grade", "level" \\
\hline & & $21 b$ & "stage", "grade", "level" \\
\hline & & 22 & "interval", "after" \\
\hline & \multirow{2}{*}{ Test results } & $24 a$ & "ROC", "AUC" \\
\hline & & $24 b$ & "confidence interval", "standard deviation", "standard error" \\
\hline & Discussion & 27 & "limitation" \\
\hline
\end{tabular}

cy articles. More than $60 \%$ of articles adhered to the ethics (69.54\%) and source of support $(63.58 \%)$ since these items were applicable to these articles.

Other items such as test method $(77.77 \%)$, test results (66.23) and analysis (55.74) were adhered by more than $50 \%$ of the articles.

Reporting adverse events (9.27\%), prespecified sample size $(10.59 \%)$, reporting the distribution of alternative diagnoses in those without target condition (17.21\%) were other items with low adherence to STARD checklist.
However, abstracts and definitions of index tests were the items that completely adhered to the STARD checklist. All of the evaluated articles were completely applicable in clinical practice.

$14.57 \%$ did not determine the study design in the article; however, $85.43 \%$ of studies specified the study design as either retrospective or prospective. Among the latter, 54.30\% were retrospective and $31.13 \%$ were prospective studies, respectively.

If an article adhered to each STARD item, 
Table 7: The characteristics of selected journals in terms of being indexed in different databases ( $y=$ yes and $n=n o), H$-index, country

\section{Journals}

\begin{tabular}{|c|c|c|c|c|c|c|}
\hline & \\
\hline Academic Radiology & $\mathrm{y}$ & $\mathrm{n}$ & $\mathrm{y}$ & $\mathrm{n}$ & 75 & United States \\
\hline Acta radiologica & $\mathrm{y}$ & $\mathrm{y}$ & $\mathrm{y}$ & $\mathrm{n}$ & 57 & United Kingdom \\
\hline American Journal of Neuroradiology & $\mathrm{y}$ & $\mathrm{y}$ & $\mathrm{y}$ & $\mathrm{y}$ & 141 & United States \\
\hline American journal of roentgenology & $\mathrm{y}$ & $\mathrm{y}$ & $\mathrm{y}$ & $\mathrm{y}$ & 159 & United States \\
\hline Annals of Nuclear Medicine & $\mathrm{y}$ & $\mathrm{y}$ & $\mathrm{y}$ & $\mathrm{n}$ & 45 & Japan \\
\hline BMC Medical Imaging & $\mathrm{y}$ & $\mathrm{y}$ & $\mathrm{y}$ & $\mathrm{n}$ & 22 & United Kingdom \\
\hline British journal of radiology & $\mathrm{y}$ & $\mathrm{y}$ & $\mathrm{y}$ & $\mathrm{n}$ & 86 & United Kingdom \\
\hline Clinical Radiology & $\mathrm{y}$ & $\mathrm{n}$ & $\mathrm{y}$ & $\mathrm{y}$ & 71 & United Kingdom \\
\hline Dentomaxillofacial Radiology & $\mathrm{n}$ & $\mathrm{y}$ & $\mathrm{y}$ & $\mathrm{n}$ & 54 & United Kingdom \\
\hline $\begin{array}{l}\text { Egyptian Journal of Radiology and } \\
\text { Nuclear Medicine }\end{array}$ & $\mathrm{y}$ & $\mathrm{n}$ & $\mathrm{n}$ & $\mathrm{n}$ & 4 & Netherlands \\
\hline Emergency Radiology & $\mathrm{y}$ & $\mathrm{y}$ & $\mathrm{y}$ & $\mathrm{n}$ & 31 & United States \\
\hline $\begin{array}{l}\text { European Journal of Nuclear Medi- } \\
\text { cine and Molecular Imaging }\end{array}$ & $\mathrm{y}$ & $\mathrm{n}$ & $\mathrm{y}$ & $\mathrm{n}$ & 106 & Germany \\
\hline European Journal of Radiology & $\mathrm{y}$ & $\mathrm{n}$ & $\mathrm{y}$ & $\mathrm{n}$ & 86 & Ireland \\
\hline European Radiology & $\mathrm{y}$ & $\mathrm{n}$ & $y$ & $\mathrm{n}$ & 118 & Germany \\
\hline $\begin{array}{l}\text { IEEE Transactions on Medical } \\
\text { Imaging }\end{array}$ & $\mathrm{y}$ & $\mathrm{n}$ & $\mathrm{y}$ & $\mathrm{n}$ & 165 & United States \\
\hline $\begin{array}{l}\text { Indian Journal of Radiology and } \\
\text { Imaging }\end{array}$ & $\mathrm{y}$ & $\mathrm{y}$ & $\mathrm{n}$ & $\mathrm{n}$ & 14 & India \\
\hline $\begin{array}{l}\text { International Journal of Radiation } \\
\text { Oncology Biology Physics }\end{array}$ & $\mathrm{y}$ & $\mathrm{y}$ & $\mathrm{y}$ & $\mathrm{n}$ & 198 & United States \\
\hline Investigative radiology & $\mathrm{y}$ & $\mathrm{y}$ & $\mathrm{y}$ & $\mathrm{n}$ & 89 & United States \\
\hline JACC: Cardiovascular Imaging & $\mathrm{y}$ & $\mathrm{y}$ & $\mathrm{y}$ & $\mathrm{n}$ & 71 & United States \\
\hline $\begin{array}{l}\text { Journal of Computer Assisted } \\
\text { Tomography }\end{array}$ & $\mathrm{y}$ & $\mathrm{y}$ & $\mathrm{y}$ & $\mathrm{n}$ & 80 & United States \\
\hline $\begin{array}{l}\text { Journal of Medical Imaging and } \\
\text { Radiation Oncology }\end{array}$ & $\mathrm{y}$ & $\mathrm{y}$ & $\mathrm{y}$ & $\mathrm{y}$ & 30 & Australia \\
\hline Journal of Ultrasound in Medicine & $\mathrm{y}$ & $\mathrm{y}$ & $\mathrm{n}$ & $\mathrm{n}$ & 67 & United States \\
\hline Korean Journal of Radiology & $\mathrm{y}$ & $\mathrm{y}$ & $\mathrm{y}$ & $\mathrm{y}$ & 38 & South Korea \\
\hline Nuclear Medicine Communication & $\mathrm{y}$ & $\mathrm{y}$ & $\mathrm{y}$ & $\mathrm{n}$ & 59 & United States \\
\hline Pediatric Radiology & $\mathrm{y}$ & $\mathrm{n}$ & $\mathrm{y}$ & $\mathrm{n}$ & 66 & Germany \\
\hline polish journal of radiology & $\mathrm{y}$ & $\mathrm{y}$ & $\mathrm{n}$ & $\mathrm{n}$ & 7 & Poland \\
\hline Practical radiation oncology & $\mathrm{y}$ & $\mathrm{y}$ & $\mathrm{n}$ & $\mathrm{n}$ & 13 & Netherlands \\
\hline radiologica medica & $\mathrm{y}$ & $\mathrm{y}$ & $\mathrm{y}$ & $\mathrm{n}$ & 34 & Italy \\
\hline Radiology & $\mathrm{y}$ & $y$ & $\mathrm{y}$ & $\mathrm{y}$ & 240 & United States \\
\hline Skeletal Radiology & $\mathrm{y}$ & $\mathrm{y}$ & $\mathrm{y}$ & $\mathrm{y}$ & 68 & Germany \\
\hline $\begin{array}{l}\text { Ultrasound in Obstetrics and Gyne- } \\
\text { cology }\end{array}$ & $\mathrm{y}$ & $\mathrm{y}$ & $\mathrm{y}$ & $\mathrm{y}$ & 106 & United Kingdom \\
\hline Total & 30 & 23 & 26 & 8 & & \\
\hline
\end{tabular}


Table 8: The number and percentage of journals which were randomly selected in terms of the country

\begin{tabular}{|c|c|c|c|c|c|}
\hline Continent & Country & Number/ Country & $\%$ & Number/ Continent & $\%$ \\
\hline America & United States & 12 & 38.71 & 12 & 38.71 \\
\hline \multirow{6}{*}{ Europe } & United Kingdom & 6 & 19.35 & \multirow{6}{*}{14} & \multirow{6}{*}{45.16} \\
\hline & Germany & 4 & 12.90 & & \\
\hline & Netherlands & 1 & 3.23 & & \\
\hline & Italy & 1 & 3.23 & & \\
\hline & Ireland & 1 & 3.23 & & \\
\hline & Poland & 1 & 3.23 & & \\
\hline \multirow{3}{*}{ Asia } & Japan & 1 & 3.23 & \multirow{3}{*}{3} & \multirow{3}{*}{9.68} \\
\hline & Korea & 1 & 3.23 & & \\
\hline & India & 1 & 3.23 & & \\
\hline Australia & Australia & 1 & 3.23 & 1 & 3.23 \\
\hline Africa & Egypt & 1 & 3.23 & 1 & 3.23 \\
\hline & Total & 31 & 100 & 31 & 100 \\
\hline
\end{tabular}

the score of 1 and if not the score of 0 were assigned to it. The total scores were normalized to 100 . The mean score of all journals of each country and each continent were calculated and shown in Figure 2.

\section{Discussion}

Diagnostic accuracy studies play a major role in promoting medical science and evolution of diagnostic methods. STARD statement was developed to help the standardization of these reports. Since then, it was always the question that how much these statements improved the reporting of these articles and how much the diagnostic accuracy studies adhered to this reporting format.

This study was intended to evaluate the adherence of diagnostic accuracy studies published in the radiology journals indexed in the Web of Science, PubMed and Scopus with STARD standard guide 2015.

As it was noted before, radiology articles were adhered to $69.45 \%$ of the STARD items. Considering that STARD statement was used since 2003 till now (12 years), it is expected to observe this adherence.
Previous studies, from 2005 to 2017, also pointed that the STARD statement was not followed properly in reporting the diagnostic accuracy studies [7, 10, 12-14, 20].

In 2005, N. Smidt et al. assessed dozens of English journals with high impact factors (4 or more). This study revealed that even in journals with high impact factor, the quality of diagnostic accuracy articles was less than normal [4]. According to Figure 2, there was not any significant difference between the score of American, European, African, Australian or Asian journals. Based on the results, the mean scores of the journals with $\mathrm{H}$ indexes more than $100(67.34 / 100)$ and the journals with $H$ indexes less than $100(68.08 / 100)$ were not different. Even the scores of lower $\mathrm{H}$ indexes obtained a non-significant more scores. This implies that high h- index journals or the journals located in advanced countries have the same quality as low $\mathrm{H}$-index or the journals in developed countries.

In a study in 2006 by Roposch et al., the adherence of the diagnostic accuracy studies regarding developmental dysplasia of the hip was evaluated. They concluded that most of 
Table 9: The number of primarily selected articles and the number of diagnostic accuracy articles

\begin{tabular}{|c|c|c|}
\hline Journals & $\begin{array}{c}\text { Number of selected } \\
\text { article }\end{array}$ & $\begin{array}{c}\text { Number of diagnostic } \\
\text { accuracy article }\end{array}$ \\
\hline Academic Radiology & 15 & 5 \\
\hline acta radiologica & 6 & 6 \\
\hline American Journal of Neuroradiology & 14 & 12 \\
\hline American journal of roentgenology & 10 & 10 \\
\hline Annals of Nuclear Medicine & 3 & 3 \\
\hline BMC Medical Imaging & 5 & 2 \\
\hline British journal of radiology & 21 & 3 \\
\hline Clinical Radiology & 12 & 5 \\
\hline Dentomaxillofacial Radiology & 9 & 3 \\
\hline Egyptian Journal of Radiology and Nuclear Medicine & 14 & 5 \\
\hline Emergency Radiology & 3 & 1 \\
\hline European Journal of Nuclear Medicine and Molecular Imaging & 9 & 8 \\
\hline European Journal of Radiology & 14 & 14 \\
\hline European Radiology & 8 & 6 \\
\hline IEEE Transactions on Medical Imaging & 3 & 1 \\
\hline Indian Journal of Radiology and Imaging & 10 & 5 \\
\hline International Journal of Radiation Oncology Biology Physics & 11 & 3 \\
\hline Investigative radiology & 16 & 2 \\
\hline JACC: Cardiovascular Imaging & 3 & 3 \\
\hline Journal of Computer Assisted Tomography & 4 & 4 \\
\hline Journal of Medical Imaging and Radiation Oncology & 8 & 7 \\
\hline Journal of Ultrasound in Medicine & 6 & 5 \\
\hline Korean Journal of Radiology & 10 & 2 \\
\hline Nuclear Medicine Communication & 7 & 7 \\
\hline Pediatric Radiology & 5 & 3 \\
\hline polish journal of radiology & 7 & 6 \\
\hline Practical radiation oncology & 7 & 1 \\
\hline radiologica medica & 6 & 3 \\
\hline Radiology & 11 & 8 \\
\hline Skeletal Radiology & 12 & 2 \\
\hline Ultrasound in Obstetrics and Gynecology & 6 & 6 \\
\hline Total & 275 & 151 \\
\hline
\end{tabular}

the articles reported just $40 \%$ of the STARD statement [8]. In the same year, Smidt et al. evaluated the published article in 12 medical medicine journals in the years 2000 and 2004 . The results of their study revealed that the studies published in 2000 reported less than $50 \%$ of the STARD items, but the studies published in 2004 reported a little more than 50\% of STARD items [6]. Again in the same year in another study by Coppus et al. which was per- 
Table 10: The number and percentage of articles that adhere to different parts of STARD standard

\begin{tabular}{|c|c|c|c|c|c|c|}
\hline section & sub group & Items & Number & $\%$ & Mean & SD \\
\hline STARD in text & & the word "STARD" used in the study & 1 & 0.66 & ---- & --- \\
\hline \multirow{5}{*}{$\begin{array}{l}\text { TITLE OR } \\
\text { ABSTRACT }\end{array}$} & \multirow{5}{*}{$\begin{array}{l}\text { title or ab- } \\
\text { stracts }\end{array}$} & 1a. Title & 149 & 98.68 & \multirow{5}{*}{97.88} & \multirow{5}{*}{2.06} \\
\hline & & 1b. Abstract & 151 & 100 & & \\
\hline & & 2. Structured Abstract & 150 & 99.34 & & \\
\hline & & 3. background & 145 & 96.03 & & \\
\hline & & 4. study objective & 144 & 95.36 & & \\
\hline \multirow{18}{*}{ Method } & study design & 5. data collection & 129 & 85.43 & ---- & ---- \\
\hline & \multirow{4}{*}{ participants } & 6. including / excluding criteria & 144 & 95.36 & \multirow{4}{*}{96.68} & \multirow{4}{*}{2.42} \\
\hline & & 7. how eligible & 150 & 99.34 & & \\
\hline & & 8. location and dates & 142 & 94.04 & & \\
\hline & & 9. patient sampling & 148 & 98.01 & & \\
\hline & \multirow{7}{*}{ Test methods } & 10a. Index test & 151 & 100 & \multirow{7}{*}{77.77} & \multirow{7}{*}{13.70} \\
\hline & & 10b. reference or gold standard & 133 & 88.08 & & \\
\hline & & 11. Reference standard rationality & 127 & 84.11 & & \\
\hline & & 12a. test positivity cut-offs of the index test & 116 & 76.82 & & \\
\hline & & 12b. test positivity cut-offs the reference standard & 96 & 63.58 & & \\
\hline & & 13a. Blind to the index test & 99 & 65.56 & & \\
\hline & & 13b. Blind to the reference standard & 100 & 66.23 & & \\
\hline & \multirow{6}{*}{ Analysis } & 14. Methods for estimating diagnostic accuracy & 138 & 91.39 & \multirow{6}{*}{55.74} & \multirow{6}{*}{38.36} \\
\hline & & $\begin{array}{l}\text { 15. How indeterminate index test or reference standard } \\
\text { results were handled }\end{array}$ & 119 & 78.81 & & \\
\hline & & 16. How missing data were handled & 56 & 37.09 & & \\
\hline & & $\begin{array}{l}\text { 17. Any analyses for distinguishing pre-specified from } \\
\text { exploratory }\end{array}$ & 28 & 18.54 & & \\
\hline & & 18a. pre-specified sample size & 16 & 10.6 & & \\
\hline & & 18b. How the sample size was chosen & 148 & 98.01 & & \\
\hline \multirow{9}{*}{ Results } & \multirow{5}{*}{ Participants } & 19. Flow of participants & 88 & 58.28 & \multirow{5}{*}{55.36} & \multirow{5}{*}{28.99} \\
\hline & & 20. Demographics of participants & 146 & 96.69 & & \\
\hline & & $\begin{array}{l}\text { 21a. Distribution of severity of disease in those with the } \\
\text { target condition }\end{array}$ & 93 & 61.59 & & \\
\hline & & $\begin{array}{l}\text { 21b. Distribution of alternative diagnoses in those without } \\
\text { the target condition }\end{array}$ & 26 & 17.22 & & \\
\hline & & 22. Time interval between index test and reference standard & 65 & 43.05 & & \\
\hline & \multirow{4}{*}{ Test results } & 23. Cross tabulation of the index test results & 122 & 80.79 & \multirow{4}{*}{66.23} & \multirow{4}{*}{38.74} \\
\hline & & 24a. Estimates of diagnostic accuracy & 145 & 96.03 & & \\
\hline & & 24b. Estimates of precision the diagnostic accuracy & 119 & 78.81 & & \\
\hline & & 25. Any adverse events & 14 & 9.27 & & \\
\hline \multirow{2}{*}{\multicolumn{2}{|c|}{ DISCUSSION }} & 26. Study limitations & 130 & 86.09 & \multirow{2}{*}{93.05} & \multirow{2}{*}{9.84} \\
\hline & & 27. Implications for practice & 151 & 100 & & \\
\hline \multirow{4}{*}{\multicolumn{2}{|c|}{ OTHER INFORMATION }} & 28. Registration number and name of registry & 1 & 0.66 & \multirow{4}{*}{35.10} & \\
\hline & & 29. Where the full study protocol can be accessed & 10 & 6.62 & & 19 \\
\hline & & 30. Sources of funding and other support; role of funders & 96 & 63.58 & & 30.49 \\
\hline & & ethics & 105 & 69.54 & & \\
\hline
\end{tabular}




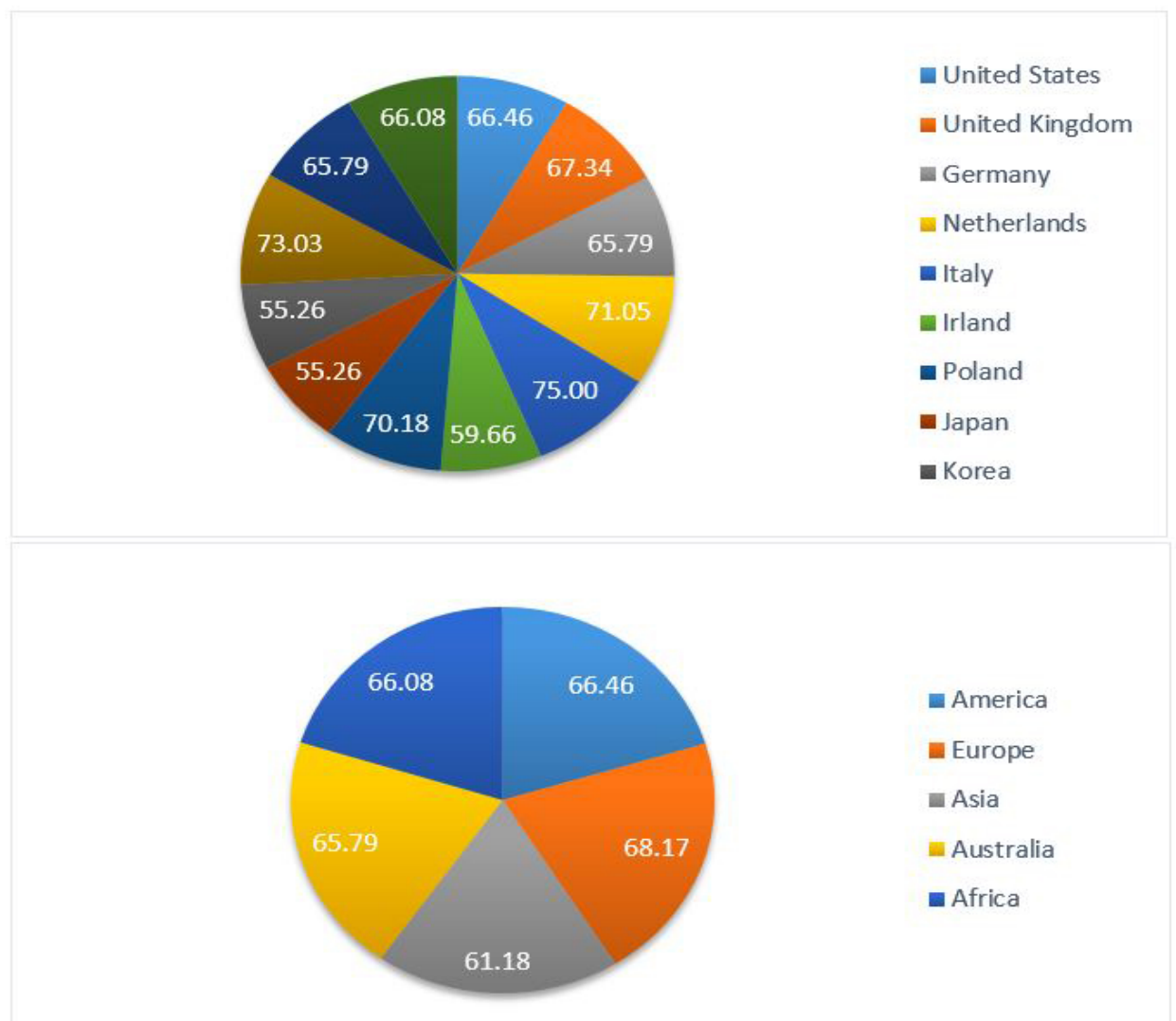

Figure 2: Up) the score of journals (/100) from each country. Down) the mean score of journals in each continent.

formed on the journals of fertility and sterility, the quality of diagnostic accuracy studies published in 1999 (before the application of STARD guideline) and the papers published in 2004 (after the application of STARD guideline) were compared. The results revealed that less than half of the diagnostic accuracy articles published in both years adhered to $50 \%$ or more items of the STARD statement [7]. Table 10 of our study shows that on average the articles adhered to $69.45 \%$ of the STARD statement, which shows an almost $40 \%$ improvement in reporting the diagnostic accuracy studies at 2015 in comparison to 2006.

In 2007, Smidt et al. published a letter to editor in order to report that the results of their study indicated only a limited number of journals which published diagnostic accuracy studies to introduce STARD statement in the instructions for authors section, and even those that do refer to STARD use varying language in their instructions. This inconsistency can be a reason for the inadherence of these studies with the standard [11]. As Smidt et al. declared in 2007, the results of this study also demonstrated that the STARD statement was not introduced to the authors in most journals. Among 31 journals of this study, only 8 journals introduced STARD statement to the authors which show that most of the authors still do not know STARD statement.

\section{Conclusion}

The adherence of radiology diagnostic accuracy studies in radiology journals (the English language articles) to STARD was more almost $70 \%$. So that the reporting of these studies improved. In order to even obtain more im- 
provements, STARD statement should be introduced in the instruction section for authors of the journals that publish diagnostic accuracy studies. The authors, reviewers and editors should be trained to implement STARD to report accurately.

The well-known items of STARD obtained more scores, but the new items which were a little unknown to the authors obtained less scores so that it is suggested to introduce these items to the authors and referees of the journals to pay more attention to them.

\section{Acknowledgment}

The authors would like to warmly thank Dr. Nasrin Shokrepoor, Dr. Zahra Bagheri and Dr. Behrooz Astaneh for their kind helps in this project.

\section{Conflict of Interest}

None

\section{References}

1. Rutjes AW, Reitsma JB, Di Nisio M, Smidt N, van Rijn JC, Bossuyt PM. Evidence of bias and variation in diagnostic accuracy studies. CMAJ. 2006;174:469-76. doi.org/10.1503/cmaj.050090. PubMed PMID: 16477057. PubMed PMCID: 1373751.

2. Bossuyt PM, Reitsma JB, Bruns DE, Gatsonis CA, Glasziou PP, Irwig LM, et al. The STARD statement for reporting studies of diagnostic accuracy: explanation and elaboration. Clin Chem. 2003:49:7-18. doi.org/10.1373/49.1.7. PubMed PMID: 12507954

3. Bossuyt PM, Reitsma JB, Bruns DE, Gatsonis CA, Glasziou PP, Irwig L, et al. STARD 2015: An Updated List of Essential Items for Reporting Diagnostic Accuracy Studies. Radiology. 2015;277:82632. doi.org/10.1148/radiol.2015151516. PubMed PMID: 26509226.

4. Smidt N, Rutjes AW, van der Windt DA, Ostelo RW, Reitsma JB, Bossuyt PM, et al. Quality of reporting of diagnostic accuracy studies. Radiology. 2005;235:347-53. doi.org/10.1148/radiol.2352040507. PubMed PMID: 15770041.

5. Siddiqui MA, Azuara-Blanco A, Burr J. The quality of reporting of diagnostic accuracy studies published in ophthalmic journals. $\mathrm{Br} J$ Ophthalmol. 2005;89:261-5. doi.org/10.1136/bjo.2004.051862. PubMed PMID: 15722299. PubMed PMCID:
1772540.

6. Smidt N, Rutjes AW, van der Windt DA, Ostelo RW, Bossuyt PM, Reitsma JB, et al. The quality of diagnostic accuracy studies since the STARD statement: has it improved? Neurology. 2006;67:792-7. doi.org/10.1212/01.wnl.0000238386.41398.30. PubMed PMID: 16966539.

7. Coppus SF, van der Veen F, Bossuyt PM, Mol BW. Quality of reporting of test accuracy studies in reproductive medicine: impact of the Standards for Reporting of Diagnostic Accuracy (STARD) initiative. Fertil Steril. 2006;86:1321-9. doi.org/10.1016/j.fertnstert.2006.03.050. PubMed PMID: 16978620.

8. Roposch A, Moreau NM, Uleryk E, Doria AS. Developmental dysplasia of the hip: quality of reporting of diagnostic accuracy for US. Radiology. 2006;241:854-60. doi.org/10.1148/radiol.2413051358. PubMed PMID: 17053199.

9. Johnston KC, Holloway RG. There is nothing staid about STARD: progress in the reporting of diagnostic accuracy studies. Neurology. 2006;67:7401. doi.org/10.1212/01.wnl.0000238980.88377.25. PubMed PMID: 16966532.

10. Johnson ZK, Siddiqui MA, Azuara-Blanco A. The quality of reporting of diagnostic accuracy studies of optical coherence tomography in glaucoma. Ophthalmology. 2007;114:1607-12. doi.org/10.1016/j. ophtha.2006.11.036. PubMed PMID: 17434589.

11. Smidt N, Overbeke J, de Vet H, Bossuyt P. Endorsement of the STARD Statement by biomedical journals: survey of instructions for authors. Clin Chem. 2007;53:1983-5. doi.org/10.1373/ clinchem.2007.090167. PubMed PMID: 17954503.

12. Wilczynski NL. Quality of reporting of diagnostic accuracy studies: no change since STARD statement publication--before-and-after study. Radiology. 2008;248:817-23. doi.org/10.1148/ radiol.2483072067. PubMed PMID: 18710977. PubMed PMCID: 2657849.

13. Fontela PS, Pant Pai N, Schiller I, Dendukuri N, Ramsay A, Pai M. Quality and reporting of diagnostic accuracy studies in TB, HIV and malaria: evaluation using QUADAS and STARD standards. PLOS One. 2009;4:e7753. doi.org/10.1371/journal.pone.0007753. PubMed PMID: 19915664. PubMed PMCID: 2771907.

14. Areia M, Soares M, Dinis-Ribeiro M. Quality reporting of endoscopic diagnostic studies in gastrointestinal journals: where do we stand on the use of the STARD and CONSORT statements? Endoscopy. 2010;42:138-47. doi. org/10.1055/s-0029-1243846. PubMed PMID: 
20140830.

15. Selman TJ, Morris RK, Zamora J, Khan KS. The quality of reporting of primary test accuracy studies in obstetrics and gynaecology: application of the STARD criteria. BMC Womens Health. 2011;11:8. doi.org/10.1186/1472-6874-11-8. PubMed PMID: 21429185. PubMed PMCID: 3072919.

16. Zintzaras E, Papathanasiou AA, Ziogas DC, Voulgarelis $M$. The reporting quality of studies investigating the diagnostic accuracy of anti-CCP antibody in rheumatoid arthritis and its impact on diagnostic estimates. BMC Musculoskelet Disord. 2012;13:113. doi.org/10.1186/1471-2474-13113. PubMed PMID: 22730931. PubMed PMCID: 3488511.

17. Korevaar DA, Wang J, van Enst WA, Leeflang MM, Hooft L, Smidt N, et al. Reporting diagnostic accuracy studies: some improvements after 10 years of STARD. Radiology. 2015;274:781-9. doi.org/10.1148/radiol.14141160. PubMed PMID: 25350641.

18. Boursier J, de Ledinghen V, Poynard T, Guechot J, Carrat F, Leroy V, et al. An extension of STARD statements for reporting diagnostic accuracy studies on liver fibrosis tests: the Liver-FibroSTARD standards. J Hepatol. 2015;62:807-15. doi. org/10.1016/j.jhep.2014.10.042. PubMed PMID: 25450206.

19. Charan J, Biswas T. How to calculate sample size for different study designs in medical research? Indian J Psychol Med. 2013;35:121-6. doi. org/10.4103/0253-7176.116232. PubMed PMID: 24049221. PubMed PMCID: 3775042.

20. Hu ZD. STARD guideline in diagnostic accuracy tests: perspective from a systematic reviewer. Ann Transl Med. 2016;4:46. PubMed PMID: 26904568. PubMed PMCID: 4740009. 